\title{
Publisher's Note: Lattice Boltzmann approach for complex nonequilibrium flows [Phys. Rev. E 92, 043308 (2015)]
}

A. Montessori, P. Prestininzi, M. La Rocca, and S. Succi

(Received 19 November 2015; published 3 December 2015)

DOI: 10.1103/PhysRevE.92.069901

PACS number(s): 47.11.-j, 47.45.Ab, 47.56.+r, 99.10.Fg

This paper was published online on 22 October 2015 with an omission in the Acknowledgments. The Acknowledgments should read as "The research has been supported by the Italian national project Hydroelectric energy by osmosis in coastal areas, PRIN 2010-2011. This work was also supported by the Integrated Mesoscale Architectures for Sustainable Catalysis (IMASC) Energy Frontier Research Center (EFRC) of the Department of Energy, Basic Energy Sciences, Award No. DE-SC0012573." The Acknowledgments have been corrected as of 20 November 2015. The Acknowledgments are incorrect in the printed version of the journal. 